\title{
Specially Corner
}

\section{My personal portable PACS station}

\section{By: Mark Haagensen}

As a radiologist with a special interest in magnetic resonance (MR) imaging and teaching, I have spent years looking for a computer solution to archive teaching cases electronically. At last I have found something way beyond my expectations.

\section{Background}

I have been digitising films for many years, initially for specific lectures using a film scanner. Recently, I have used a digital camera selecting images on the 'run' during a busy working day for use in general teaching. Although the image quality is good, I have ended up with enormous files of digital images, many unopened, which often have incomplete demographics and an uncertain diagnosis. Consequently, preparing a presentation which requires searching through and transferring poorly catalogued JPEG (Joint Photographic Experts Group) images into a PowerPoint (Microsoft Office, Microsoft Corporation, Redmond, USA) presentation is very time consuming. How much better to have all the images already organised into electronic teaching files!

Teaching library programs available off the Internet, have been reviewed. ${ }^{1}$ Many of these are very good but it is still a laborious task to enter JPEG images with history and findings into these databases, and on top of that the retrieval of CT or MR teaching cases from workstations via $\mathrm{CD}$ or 'memory sticks' is rather cumbersome.

Over the last few years many DICOM (Digital Imaging and Communications in Medicine standard) software viewers have become available off the Internet. ${ }^{2,3}$ Scanner vendors will also supply a very basic DICOM viewer when exporting images to $\mathrm{CD}$, which is of little use. My electronic teaching 'project' remained in a shambles until a year ago.

\section{Changing systems}

At that time I had become thoroughly frustrated by 'Windoze' (term of endearment for the operating system on PCs) and when my laptop screen expired, I decided to switch computer system and bought an Apple laptop computer, 'MacBookPro' (Apple Computers Inc., Cupertino, $\mathrm{Ca}$, USA). I will avoid going into the difficult comparison of two such different packages as an Apple Computer with its integrated software and the Microsoft Windows software which has to be able to run on a variety of different quality machines. Suffice to say, the Mac operating system does not need antivirus programs, and rarely if a program does 'hang', you can force it to quit without the computer crashing.

While showing off my new Mac, I got to hear about a visiting UK radiologist who was teaching, using his Mac laptop as a workstation! I tracked him down quickly for more details.

\section{Eureka!}

The program is called Osirix, open source software (OSS) downloadable free off the Internet at http://homepage.mac.com/rossetantoine/osirix, but it only runs on the Apple platform (operating system X - OS X) for the moment. ${ }^{4,5}$

This is truly an amazing DICOM and PACS program. Osirix will accept DICOM CDs from all vendors, and if it doesn't 'see' the DICOM folder automatically, exploring the CD's hidden directories is simple. This is not possible on our workstations, which run on a Windows platform, but do not allow access to the Windows programs, presumably for 'security' reasons. This restriction of access I find very frustrating, especially considering the hefty price of these workstations. It would be much more efficient to run the viewing software in open integration with PowerPoint, web browser for quick internet searches, and e-mail for sending selected images to colleagues or referring physicians. This is however possible with Osirix.

\section{The personal PACS gets underway}

Fortunately, I have a connection point for our Ethernet at my desk linking scanners and workstations. Sending cases straight to my laptop via Ethernet was fairly easy with the help of the MR technicians, who needed to set up the correct addresses of my laptop on the MR console and later CT workstation as well. These technicalities are described on a congress poster which can be downloaded from the web. ${ }^{6}$ It was not long before I was reporting MR studies off my laptop, increasing my efficiency considerably compared with my previous film reading.

Picture quality is superb and image handling fast. In Osirix the images are available instantly; it can view multiple sequences in flexible windows and relates them automatically to each other, i.e. while scrolling through an axial stack, the current slice is indicated by a line on the sagittal and coronal views, and if one double clicks on a lesion its position is immediately indicated on the other open sequences of the study.

Old and new CT studies can be compared in both the axial and coronal projections which is not possible on our conventional workstation. One can subtract and fuse images easily with 'drag and drop', for example fusing an MR angio sequence with a FLAIR sequence not only slice by slice, but also in 3D. Calculating organ or lesion volume is simple and when drawing ROIs (region of interest) Osirix has an amazing 'repulsor tool' for pushing the line into position.

Of course the usual data manipulations are available, such as MPR (multi-planar reconstructions), and MIP (maximum intensity projections), but also more complex processes such as volume and surface rendering, which are facilitated by various cutting and seeding tools as well as CLUT (colour lookup table) editors.

\section{The Osirix user group}

The Osirix team, led by a radiologist and software developer Antoine Rosset, are developing and improving the program constantly, getting feedback from the User group, which now numbers over 10000 worldwide. Joining this group will give you access to a regular stream of e-mails (questions and answers, requests and announcements of new downloadable upgrades) from all over the world, and quite entertaining sometimes. The use of OSS is new to radiology, but judging from the quality of Osirix and the enthusiasm, it is here to stay (for more detail and history on OSS, search www.wikipedia.org, which also has a manual on Osirix).

\section{Who can benefit from Osirix}

For clinicians, Osirix represents a cost-effective solution, enabling them to have access and the ability to manipulate their imaging studies for better patient management. The DICOM viewers distributed with the patients' CDs are very basic without any facility to manipulate the dataset in detail.

For radiologists, Osirix represents a very attractive, economical solution, enabling them to have an extra or personal workstation, which will integrate with a pre-existing PACS or just your workstations. 
Osirix is particularly useful when conducting academic meetings, e.g. for our weekly neuroradiological combined meeting, the co-ordinator receives cases for discussion the day before on CD in DICOM format. Videoprojection of Osirix is in real-time and interactive and best done by somebody with a little experience of the program. The large image display promotes audience participation and learning. Osirix runs smoothly on the laptop with no restrictions making it very convenient. However additional RAM up to 2 gigabytes is generally recommended.

Video projection of Osirix is also very useful in tutorials for radiology registrars. Simulating the work situation with a PACS station and giving the candidate the original dataset to examine, seems the proper way to go. Using an OSS program(s) would be inexpensive and fairer to exam candidates by not favouring a particular vendor's workstation, and Osirix is easy to use for general manipulations. Although Osirix only runs on the Apple OS at the moment, the new Apple computers can also run Microsoft Windows for an alternative viewer to Osirix, making Apple Mac the more flexible solution.

In the case of formal lecture presentations, Osirix again comes to the rescue. You can view your entire teaching case on the one hand, while making your presentation on the other, and the DICOM image you want to use can be 'dragged and dropped' into the 'slide', where it is automatically converted to a JPEG format. It does the same for any $3 \mathrm{D}$ or fusion images. If you need a cine, you export the series from Osirix in QuickTime format (Apple Computers Inc.) to 'desktop' and drop it into your slide.

\section{Osirix has more}

Briefly, Osirix has many other features such as a DICOM print function to laser printer, query and retrieve function from other nodes on the PACS, export in DICOM either to file or back to the PACS server of secondary generated images, report function including DICOM structured report with plans for word recognition as a plug-in in the future. However, I haven't had the opportunity or necessity to check out all the complex PACS features. Lastly, one of the most impressive and powerful functions is the $4 \mathrm{D}$ player. This involves $3 \mathrm{D}$ plus the added dimension of time; for example I tested a new super-fast contrast-enhanced MR angiogram sequence supplied by a vendor, consisting of 40 slices in 3 seconds as a volume repeated over 9 dynamics. Using the $4 \mathrm{D}$ player you can view the cine run as a MIP, and simultaneously rotate it as a 3D volume! Osirix can also do $5 \mathrm{D}$, which is the further addition of a functional parameter, such as Positron Emission Tomography (PET). There is a cine of a $5 \mathrm{D}$ study downloadable from the Osirix website (http://homepage.mac.com/rossetantoine/ osirix).

After all that - yes I did find my little teaching database as well. A subgroup of the Osirix team has made a small OSS program called Xphile modelled on the iTunes program, which can be downloaded free from http://homepage.mac.com/d2p/xphile. It integrates with Osirix making data capture easier. The need for a teaching file seems less urgent after Osirix, which has 'albums' and search functions plus report facility enough for a DICOM teaching library for the moment.

As mentioned, I have not explored Osirix to its fullest as yet, and other appealing features are teleradiology, teleconferencing and connectivity to iPod (Apple Computers Inc). ${ }^{7}$

\section{Conclusion}

Osirix is a very powerful and fast PACS and DICOM program freely available off the Internet. It is an Open Source program without any guarantees, but where one relies on support from a large user group of similarminded people with a large pool of experience. Support companies are emerging that sell support and hardware for a PACS entirely based on Osirix, which in their hands has been FDA-approved.

Osirix has made great impact on my teaching and efficiency as a radiologist. It only runs on Apple computers, but 'switching' is highly recommended as well.

1. Scarsbrook AF, Foley PT, Perriss RW, Graham RNJ. Radiologica digital teaching file development: an overview. Clin Radiol 2005; 60: 831-837.

2. Escott EJ, Rubinstein D. Free DICOM image viewing and processing software for your desktop computer: What's available and what it can do for you. Radiographics 2003; 23: 1341-1357.

3. Escott EJ, Rubinstein D. Free DICOM image viewing and processing software for the Macintosh Computer: What's available and what it can do for you. Radiographics 2004; 24:1763-1777.

4. Ratib O, Rosset A. Open-source software in medical imaging: development of OsiriX. International Journal of Computer Assisted Radiology and Surgery 2006; 1:187-196.

5. Rosset A, Spadola L, Ratib O. OsiriX. An open-source software for navigating in multidimensional DICOM images. J Digit Imaging 2004; 17: 205-216

6. Rosset A, Ratib O, Heuberger J, Tao W. How to integrate your personal computer in a PACS environment. http://homepage. mac.com/rossetantoine/osirix/PosterRSNA2005.jpg

7. Rosset C, Rosset A, Ratib O. General consumer communication tools for improved image management and communication in medicine. J Digit Imaging 2005; 18: 270-279.

\section{Congrats}

\section{By: Mala Modi}

Congrats and best wishes to my friends and colleagues, Dr Ashwin Hurribance who is the new president of the College of Radiology of South Africa, and Professors Savvas Andronikou and Victor Mngomezulu who are now at the helm of the University of Stellenbosch and Medunsa respectively. It would be remiss of me not to point out that all of them hailed from Wits University.

A further congrats to Savvas on being awarded the Derek Harwood scholarship. This is just one of two scholarships awarded by the RSNA on an annual basis. The scholarship is worth 10000 dollars, and enables Savvas to study at an American institution of his choice. He is presently at the Columbia University Medical Centre in the FMRI Centre and the Children's Hospital Neuroimaging Centre. He is learning FMRI under the supervision of Joy Hirsch and Bill Millar, and he is also busy assisting in the set-up of the DTI (diffusion tensor imaging) services there.

He will also be visiting Cornell to learn more detailed DTI, and is an invited speaker at Salt Lake City, Utah to talk on TB in children.

Well deserved and best wishes!

\section{Conference news}

\section{By: Mala Modi}

\section{Paediatric mini-conference}

Savvas will share his experiences and research findings with us next year (March 2008) when Professor Sheldon Wiebe, a paediatric radiologist from Saskatoon, Canada, will be in South Africa. We will be hosting a joint paediatric mini-conference in Johannesburg, and possibly other centres. Details will be forwarded to all via the College of Radiology and the RSSA who will be approached for financial and organisational assistance.

\section{Neuroradiology mini-conference}

A big thank you to Professor Jonathan Kleefield of the Neuroradiology Department of Harvard Medical School. Jonathan was the sole, tireless, and impressive speaker at the neuroradiology mini-conference that was held at Johannesburg Hospital in March this year.

Thank you to Robert Makhubedu of Toshiba and Mike Botha of Bracco for assisting with this endeavour. It is good to see the public sector receiving some of the goodwill from our trade partners. 